\title{
Electronegatividade: Uma Chave Para a Resolução Das Dificuldades De Aprendizagem Em Química*
}

\author{
Rute Amadeu ${ }^{1}$ e João Paulo Leal ${ }^{1,2}$
}

\begin{abstract}
A electronegatividade é um dos conceitos mais profícuos dentro da Química. Nesta contribuição começa-se por apresentar uma breve evolução histórica do conceito de electronegatividade. De seguida, sugere-se onde poderia ser introduzida nos actuais curricula e como poderia ser usada como fio condutor para o ensino/aprendizagem da Química.
\end{abstract}

Desde o seu aparecimento, a electronegatividade deu origem a uma área de investigação que continua em grande actividade, não sendo por isso um conceito datado ou ultrapassado. É também um conceito transversal a várias áreas da Química e portanto poderia ser usado como fio condutor para o ensino/aprendizagem da Química ao longo do $3^{\circ}$ ciclo do Ensino Básico e do Ensino Secundário. Nesta breve contribuição pretendemos apenas mostrar a exequibilidade desta estratégia. Começaremos por apresentar uma breve evolução histórica do conceito de electronegatividade, mostrando que pode ser abordada de modo bastante conciso. No ensino básico e secundário apenas é apresentada, e de modo demasiado básico, a escala de Pauling. De seguida, sugeriremos onde poderia o conceito ser introduzido nos actuais curricula e ainda como pedagogicamente poderia ser abordado.

\section{Evolução Histórica do Conceito DE ELECTRONEGATIVIDADE}

O conceito de electronegatividade foi introduzido por J.J. Berzelius, em 1811, que o definiu como sendo a capacidade que um átomo tem para atrair para si os electrões [1]. Linus Pauling aprofundou os estudos de Berzelius

* Baseado numa tese de Mestrado apresentada na Faculdade de Ciências da Universidade de Lisboa.

Departamento de Química e Bioquímica, Faculdade de Ciências, Universidade de Lisboa, 1149-016 Lisboa

Unidade de Ciências Químicas e Radiofarmacêuticas, Instituto Tecnológico e Nuclear, 2686-953 Sacavém e, no ano de 1931, propôs a primeira escala prática de electronegatividade que foi obtida por cálculo matemático [2]. Pauling começou por estabelecer o valor quatro como sendo o valor de electronegatividade para o átomo de flúor. A partir desse valor, calculou os valores para os restantes elementos da Tabela Periódica, através de uma equação matemática que relacionava a média aritmética da energia de ligação dos átomos envolvidos na ligação química com os respectivos valores de electronegatividade (equação 1);

$\left|\chi_{A}-\chi_{B}\right|=0,208 \sqrt{E_{A-B}-1 / 2\left(E_{A-A}+E_{B-B}\right)}$

onde $\left|\chi_{A}-\chi_{B}\right|$ é a diferença de electronegatividade entre $\chi_{A}$ e $\chi_{B}$; $E_{A-B}$ é a energia de ligação na espécie $A-B ; E_{A-A}$ é a energia de ligação na espécie homonuclear $A-A$ e $E_{B-B}$ é a energia de ligação da espécie homonuclear B-B, todas estas expressas em $\mathrm{kcal} / \mathrm{mol}$.

Apesar de a escala original de electronegatividade proposta por Pauling se basear na média aritmética dos valores das energias de ligação dos átomos, Pauling verificou que era mais rigoroso utilizar uma média geométrica dos valores dessas mesmas energias ao invés de uma média aritmética, o que o obrigou a fazer alguns ajustes na sua primeira escala de electronegatividade.

A equação matemática que permite calcular os valores de electronegatividade dos elementos, a partir da média geométrica dos valores das energias de ligação, passa então a ser a equa- ção 2 em que as grandezas têm o mesmo significado que anteriormente.

$\left|\chi_{A}-\chi_{B}\right|=0,183 \sqrt{E_{A-B}-\left(E_{A-A} \times E_{B-B}\right)^{1 / 2}}$

Os valores de electronegatividade tornaram-se extremamente úteis na previsão do carácter químico das ligações, podendo ser usados para prever de modo aproximado o grau de ionicidade de uma ligação química (equação 3), onde $\chi_{A}$ é a electronegatividade do átomo $\mathrm{A}$ e $\chi_{B}$ a electronegatividade do átomo $B$ [3].

$\%$ Carácter Iónico $=1-e^{-\frac{1}{4}\left(\chi_{A}-\chi_{B}\right)^{2}}$

Numa tentativa de aperfeiçoar o conceito de electronegatividade introduzido por Linus Pauling no ano de 1932, vários cientistas dedicaram-se à criação de escalas de electronegatividade, sendo algumas delas concordantes com a de Pauling. Outras, por seu lado, divergiam bastante quer na ideia subjacente quer nos valores de electronegatividade obtidos.

Em 1934, Robert S. Mulliken sugeriu que a electronegatividade dos elementos deveria ser calculada através da média aritmética do valor da primeira energia de ionização e da afinidade electrónica [4] (equação 4 ), onde $\chi^{M}$ seria a electronegatividade, $\Delta E$ a afinidade electrónica e $\Delta E_{y}$ a energia de ionização (actualmente e, de acordo com a nomenclatura IUPAC, a energia de ionização representa-se por I).

$\chi^{M}=\frac{\Delta E+\Delta E_{y}}{2}$ 
Esta teoria divergia em alguns aspectos da proposta por Linus Pauling, uma vez que, ao contrário da escala de Pauling que se baseia em propriedades dos átomos em interacção (energias de ligação), a escala de Mulliken baseia-se em propriedades intrínsecas de cada átomo (energia de ionização e afinidade electrónica). $\mathrm{Na}$ escala de Mulliken, o néon é o elemento mais electronegativo da Tabela Periódica, ao passo que Linus Pauling, não considerava os gases raros, e apontava o flúor como o elemento com maior electronegatividade. No entanto e, apesar de os valores numéricos serem diferentes, é possível estabelecer uma relação entre as duas escalas.

Em 1946, Gordy introduziu uma nova escala de electronegatividade que se baseava na força de atracção electrostática entre o núcleo de um átomo e os electrões de valência [5]. A electronegatividade seria dada pela equação 5 , onde $r$ é a distância entre o electrão e o núcleo, $e$ a carga do electrão e $Z_{\text {eff }}$ a carga nuclear efectiva ${ }^{1}$.

$$
\chi=\frac{e \times Z_{e f f}}{r}
$$

Outros trabalhos relevantes na área da electronegatividade, foram os de Walsh que concluiu que a electronegatividade do átomo de carbono dependia do seu estado de hibridação [6] e, os de Sanderson, que sugeriu que a electronegatividade de um elemento dependia do estado de oxidação do átomo e, consequentemente, da sua estabilidade radial [7]. Sanderson definiu uma relação matemática para calcular os diversos valores de electronegatividade (equação 6), onde $\chi$ é o valor de electronegatividade na escala de Pauling e $S R$ a estabilidade radial [8].

$$
\chi^{1 / 2}=0,21 S R+0,77
$$

Em 1958 Allred e Rochow definiram a electronegatividade em função da carga nuclear efectiva e do raio covalente dos átomos, tal como Gordy tinha sugerido anteriormente, tendo, no entanto, feito algumas alterações à expressão matemática utilizada por Gordy [9]. A força de atracção electrostática foi então definida pela equação 7 , onde $r$ é a distância entre o electrão e o núcleo, $e$ a carga do electrão e $Z_{\text {eff }}$ a carga nuclear efectiva.

$F=\frac{e^{2} \times Z_{\text {eff }}}{r^{2}}$

Esta nova definição representa uma electronegatividade absoluta, porque não foi necessário fixar um valor para a electronegatividade de um elemento como referência; diferindo assim dos valores de Pauling que eram valores relativos a um valor inicialmente definido. No entanto, as duas escalas podem ser relacionadas através da equação 8, tendo as grandezas os significados anteriormente referidos ( sendo $\chi$ a electronegatividade de Pauling).

$$
\chi=0,359 \times \frac{Z_{e f f}}{r^{2}}+0,744
$$

Allred e Rochow mencionaram ainda que, atendendo a que a capacidade de um átomo, numa molécula, de atrair electrões depende da combinação das orbitais utilizadas na ligação e, como a maioria dos elementos possui mais que um tipo de hibridação, torna-se mais rigoroso falar em termos de electronegatividade orbital do que em electronegatividade de um elemento.

Como exemplo das várias escalas já referidas, apresenta-se na Tabela 1 os valores de electronegatividade dos vários elementos segundo Pauling (a preto), Mulliken (a azul), Sanderson (a verde) e Allred-Rochow (a vermeIho). Através da análise desta tabela, é possível verificar que a escala de electronegatividade que mais se aproxima da escala proposta por Pauling é a escala de Sanderson e a que apresenta valores mais díspares é a escala de Mulliken. É importante referir que, na Tabela 1, apenas se destacam os valores de Pauling, Mulliken, Sanderson e Allred-Rochow por estes serem considerados os pioneiros no estudo deste conceito.

Após o estudo de Allred-Rochow, Gordy definiu electronegatividade para um átomo neutro, numa molécula estável, como sendo o potencial em função da distância do raio covalente e da carga efectiva do núcleo. Assim, segundo Gordy, o potencial da ligação covalente é considerado como sendo uma medida absoluta de electronegativida- de sendo calculado pela equação 9 , onde $r$ é a distância entre o electrão e o núcleo, $e$ a carga do electrão e $n$ o número de electrões de valência [9].

$\chi_{1}=\frac{0,5 \times e \times(n+1)}{r}$

Frequentemente esta fórmula é apresentada com as constantes alteradas (equação 10) para que os seus valores sejam comparáveis com os obtidos pela escala de Pauling.

$$
\chi_{2}=0,31 \times \frac{(n+1)}{r}+0,50
$$

Pritchard e Skinner reviram a relação estabelecida por Gordy e utilizaram o método de Plater para determinar os valores da carga nuclear efectiva. Contudo, tornou-se necessário introduzir diferentes constantes na equação 10 , consoante o período da tabela periódica em que se encontram os elementos.

Em 1962, foi proposta uma nova definição de electronegatividade por Hinze, Whitehead e Jaffé, que se baseva nos trabalhos de Iczkowski e Margrave [10]. Segundo Jaffé e os seus colaboradores, a electronegatividade de um átomo dependia da natureza da orbital envolvida na ligação química bem como da ocupação dessa orbital. Esta definição assemelha-se bastante à proposta por Mulliken e pode ser traduzida através da equação 11 , onde $E(n)$ representa a energia do átomo para um dado estado de valência e $n$ representa a ocupação dessa mesma orbital [10].

$$
\chi=\frac{\partial E(n)}{\partial n}
$$

Por volta de 1965, James E. Huheey dedicou-se ao estudo da electronegatividade de grupos, tendo utilizado um método análogo ao de Jaffé. A definição de electronegatividade aparentemente mais simples foi a proposta por L. C. Allen, no ano de 1989. Segundo Allen, a electronegatividade estava relacionada com a energia média dos electrões de valência num átomo livre (equação 12 ), onde $\chi_{\text {spec }}$ é o valor de electronegatividaae espectroscópica, $m$ o número de electrões na orbital $\mathrm{p}, n$ o número de electrões na orbital s, $\varepsilon_{S}$ a energia de ionização da orbital s e $\varepsilon_{p}$ a energia de ionização 


\begin{tabular}{|c|c|c|c|c|c|c|c|c|c|c|c|c|c|c|c|c|c|c|}
\hline Grupo & 1 & $\underline{2}$ & $\underline{3}$ & 4 & $\underline{5}$ & $\underline{6}$ & 1 & $\underline{8}$ & $\underline{9}$ & 10 & 11 & 12 & $\underline{13}$ & 14 & $\underline{15}$ & $\underline{16}$ & 17 & $\underline{18}$ \\
\hline \multicolumn{19}{|l|}{ Periodo } \\
\hline 1 & $\begin{array}{c}\frac{\mathrm{H}}{2,1} \\
3,06 \\
2,31 \\
2,20\end{array}$ & & & & & & & & & & & & & & & & & He \\
\hline$\underline{2}$ & $\begin{array}{c}\frac{\underline{L i}}{1,0} \\
1,28 \\
0,86 \\
0,97\end{array}$ & $\begin{array}{c}\frac{B e}{1,5} \\
1,99 \\
1,61 \\
1,47\end{array}$ & & & & & & & & & & & $\begin{array}{c}\frac{\mathrm{B}}{2,0} \\
1,83 \\
1,88 \\
2,01\end{array}$ & $\begin{array}{c}\frac{c}{2,5} \\
2,67 \\
2,47 \\
2,50\end{array}$ & $\begin{array}{c}\frac{\mathrm{N}}{3,0} \\
3,08 \\
2,93 \\
3,07\end{array}$ & $\begin{array}{c}\underline{0} \\
3,5 \\
3,21 \\
3,46 \\
3,50\end{array}$ & $\begin{array}{c}\frac{F}{4,0} \\
4,42 \\
3,92 \\
4,10\end{array}$ & $\begin{array}{l}\underline{\mathrm{Ne}} \\
4,60\end{array}$ \\
\hline$\underline{3}$ & $\begin{array}{c}\frac{\mathrm{Na}}{0,9} \\
1,21 \\
0,85 \\
1,01\end{array}$ & $\begin{array}{c}\frac{\mathrm{Mg}}{1,2} \\
1,63 \\
1,42 \\
1,23\end{array}$ & & & & & & & & & & & $\begin{array}{c}\frac{\mathrm{Al}}{\mathbf{1}, 5} \\
1,37 \\
1,54 \\
1,47\end{array}$ & $\begin{array}{c}\frac{S i}{1,8} \\
2,03 \\
1,74 \\
1,74\end{array}$ & $\begin{array}{c}\frac{P}{2,1} \\
2,39 \\
2,16 \\
2,06\end{array}$ & $\begin{array}{c}\frac{\mathrm{s}}{2,5} \\
2,65 \\
2,66 \\
2,44\end{array}$ & $\begin{array}{c}\frac{\mathrm{Cl}}{3,0} \\
3,54 \\
3,28 \\
2,83\end{array}$ & $\begin{array}{l}\text { Ar } \\
3,36 \\
3,92\end{array}$ \\
\hline$\underline{4}$ & $\begin{array}{c}\underline{K} \\
0,8 \\
1,03 \\
0,74 \\
0,91\end{array}$ & $\begin{array}{c}\frac{\mathrm{Ca}}{1,0} \\
1,30 \\
1,06 \\
1,04\end{array}$ & $\begin{array}{l}\frac{S c}{1,3} \\
1,09 \\
1,20\end{array}$ & $\frac{T i}{1,5}$ & $\frac{\underline{v}}{1,6}$ & $\frac{c r}{1,6}$ & $\frac{M n}{1,5}$ & $\frac{\mathrm{Fe}}{1,8}$ & $\frac{C_{0}}{1,9}$ & $\frac{\mathrm{Ni}}{1,8}$ & $\frac{\mathrm{Cu}}{1,9}$ & $\begin{array}{l}\frac{\mathrm{Zn}}{1,6} \\
1,86 \\
1,66\end{array}$ & $\begin{array}{c}\frac{\mathrm{Ga}}{\mathbf{1 , 6}} \\
1,34 \\
2,10 \\
1,82\end{array}$ & $\begin{array}{c}\frac{G e}{1,8} \\
1,95 \\
2,31 \\
2,02\end{array}$ & $\begin{array}{c}\frac{A s}{2,0} \\
3,26 \\
2,53 \\
2,20\end{array}$ & $\begin{array}{c}\frac{\mathrm{Se}}{2,4} \\
2,51 \\
2,76 \\
2,48\end{array}$ & $\begin{array}{c}\frac{\mathrm{Br}}{3,0} \\
3,24 \\
2,96 \\
2,74\end{array}$ & $\begin{array}{l}\underline{\mathrm{Kr}} \\
2,98 \\
3,17\end{array}$ \\
\hline$\underline{5}$ & $\begin{array}{c}\frac{\mathrm{Rb}}{\mathrm{0}, 8} \\
0,99 \\
0,70 \\
0,89\end{array}$ & $\begin{array}{c}\frac{\mathrm{Sr}}{1,0} \\
1,21 \\
0,96 \\
0,99\end{array}$ & $\begin{array}{l}0,98 \\
1,11\end{array}$ & $\frac{\underline{z} r}{1,4}$ & $\frac{\mathrm{Nb}}{1,6}$ & $\frac{\mathrm{Mo}}{1,8}$ & $\frac{\mathrm{Tc}}{1,9}$ & $\frac{\mathrm{Ru}}{2,2}$ & $\frac{R h}{2,2}$ & $\frac{\mathrm{Pd}}{2,2}$ & $\frac{\mathrm{Ag}}{1,9}$ & $\begin{array}{l}\frac{\mathrm{Cd}}{1,7} \\
1,73 \\
1,46\end{array}$ & $\begin{array}{c}\frac{\ln }{1,7} \\
1,30 \\
1,88 \\
1,49\end{array}$ & $\begin{array}{l}\frac{S n}{1,8} \\
1,83 \\
2,02 \\
1,72\end{array}$ & $\begin{array}{c}\frac{S b}{1,9} \\
2,06 \\
2,19 \\
1,82\end{array}$ & $\begin{array}{c}\frac{T e}{2,1} \\
2,34 \\
2,34 \\
2,01\end{array}$ & $\begin{array}{c}\frac{1}{1} \\
2,5 \\
2,88 \\
2,50 \\
2,21\end{array}$ & $\begin{array}{l}\underline{X e} \\
2,59 \\
2,63\end{array}$ \\
\hline$\underline{6}$ & $\begin{array}{l}\frac{C S}{C, 7} \\
0,69 \\
0,86\end{array}$ & $\begin{array}{c}\mathrm{Ba} \\
0,9 \\
0,93 \\
0,97\end{array}$ & * & $\frac{\mathrm{Hf}}{1,3}$ & $\frac{\mathrm{Ta}}{1,5}$ & $\frac{w}{1,7}$ & $\frac{R e}{1,9}$ & $\frac{O s}{2,2}$ & $\frac{\underline{I r}}{2,2}$ & $\frac{P t}{2,2}$ & $\frac{A u}{2,4}$ & $\begin{array}{c}\frac{\mathrm{Hg}}{1,9} \\
1,92 \\
1,44\end{array}$ & $\begin{array}{c}\frac{T I}{1,8} \\
1,96 \\
1,44\end{array}$ & $\begin{array}{c}\frac{\mathrm{Pb}}{1,9} \\
2,01 \\
1,55\end{array}$ & $\begin{array}{c}\frac{B i}{1,9} \\
2,06 \\
1,67\end{array}$ & $\frac{P_{0}}{2,0}$ & $\frac{A t}{2,2}$ & $\underline{R n}$ \\
\hline 7 & $\frac{\mathrm{Fr}}{0,7}$ & $\frac{\mathrm{Ra}}{0,9}$ & ** & $\underline{R f}$ & $\underline{D b}$ & $\underline{\mathrm{sg}}$ & Bh & $\underline{\mathrm{Hs}}$ & $\underline{M t}$ & $\underline{\text { Ds }}$ & $\underline{\mathrm{Rg}}$ & $\underline{\text { Uub }}$ & Uut & $\underline{\text { Uuq }}$ & Uup & $\underline{\text { Uuh }}$ & $\underline{\text { Uus }}$ & $\underline{\text { Uno }}$ \\
\hline
\end{tabular}

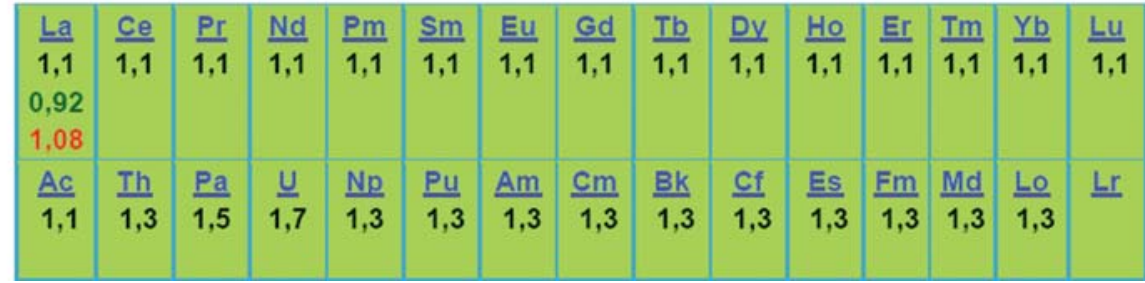

da orbital $p[11]$

$$
\chi_{\text {spec }}=\frac{m \varepsilon_{p}+n \varepsilon_{s}}{m+n}
$$

Como os valores de electronegatividade de Allen podem ser determinados directamente por métodos espectroscópicos, estes valores são muitas vezes designados por valores de electronegatividade espectroscópica.

O método de Allen permite determinar a electronegatividade de inúmeros elementos, inclusivamente daqueles que não poderiam ser determinados por nenhum outro método e dos quais se pode destacar o frâncio, cujo valor de electronegatividade, nesta escala, é de 0,67 . Contudo, este método também apresenta algumas limitações uma vez que não permite calcular a electronegatividade dos elementos dos blocos $d$ e $f$. Actualmente, a electronegatividade dos átomos continua a ser um tema bastante estudado pela comunidade científica, o que é facilmente comprovado pelo elevado número de citações dos artigos relativos a este tema; pelo que, para além das escalas aqui referidas, muitas outras foram já definidas ou refinamentos foram feitos [12] e prevê-se que muitas outras possam vir a surgir.

\section{Linhas Gerais Do Programa De ENSINO}

Pretende-se aqui dar uma noção, ainda que muito resumida, de como os actuais programas abordam a Química (ou os vários temas dentro da Química) no $3^{\circ}$ ciclo do Ensino Básico e no Ensino Secundário, de modo a mais facilmente enquadrar o restante do texto.

No $7^{\circ}$ ano de escolaridade, os alunos iniciam o seu estudo no mundo da Química começando por estudar a constituição do mundo material [13]. No $8^{\circ}$ ano de escolaridade o programa de Físico-Química, e o da Física 
em particular, dedica-se quase exclusivamente ao estudo de óptica e acústica. No que tange à unidade de Química, que geralmente é leccionada apenas no terceiro período, é feita apenas uma mera referência aos tipos de reacções químicas, à velocidade das reacções e à representação das reacções químicas [13].

No $9^{\circ}$ ano de escolaridade, na unidade de Química é efectuada uma abordagem ao conceito de estrutura atómica, tabela periódica e propriedades das substâncias, finalizando com o conceito de ligação química [13].

No $10^{\circ}$ ano de escolaridade do programa de Física e Química A, na unidade 1, "Das estrelas ao átomo", inicia-se o estudo da tabela periódica do qual faz parte a descrição da estrutura da tabela periódica; posição dos elementos na tabela periódica e respectivas configurações electrónicas; variação do raio atómico e da energia de ionização; propriedades dos elementos e das substâncias elementares. É, ainda, feita uma abordagem aos modelos de ligação química e da geometria das moléculas que existem na troposfera [14].

No $11^{\circ}$ ano de escolaridade do programa de Física e Química A, é feita uma análise muito aprofundada sobre a indústria do amoníaco, o que implica um estudo minucioso acerca desta molécula [15].

No $12^{\circ}$ ano de escolaridade do Curso de Ciências e Tecnologia, o programa consiste basicamente no estudo de: metais e ligas metálicas, reacções dos metais, combustíveis fósseis e a crise de energia, ligação química e estrutura dos hidrocarbonetos e outros compostos orgânicos e plásticos, vidros e novos materiais [16].

Através de uma breve análise dos programas é possível verificar que o conceito de electronegatividade poderia ser introduzido logo no $9^{\circ}$ ano de escolaridade, aquando do estudo das ligações químicas e ser usado como fio condutor para os posteriores temas de Química. No entanto, o conceito de electronegatividade só é introduzido, e de modo apenas marginal, no $12^{\circ}$ ano de escolaridade, ou seja, decor- ridos cinco anos desde que os alunos iniciam o seu estudo no vasto mundo da Química.

Acresce ainda que os alunos que não optem no final do $3^{\circ}$ ciclo por ter a disciplina de Física e Química A, ou optem por seguir um outro curso que não o de Ciências e Tecnologia, nunca abordarão o conceito de electronegatividade durante o seu percurso escolar.

\section{A Electronegatividade No Ensino BÁsico E SeCundárIo}

Na área da educação e, em particular, ao nível do ensino básico e secundário, continua a utilizar-se somente a definição de electronegatividade de Linus Pauling, independentemente de terem sido inúmeros os estudos inerentes a este conceito. É muito pouco frequente encontrar num manual escolar outra definição de electronegatividade que não a de Pauling. Paralelamente a esta visão algo limitada, também é possível verificar que existe um certo desfasamento entre a altura em que é abordado o conceito de electronegatividade e a altura em que seria desejável e, até mais vantajoso, a sua inserção.

É possível verificar, através da Figura 1 (ver página seguinte), que o conceito podia, e na nossa opinião devia, ser introduzido com facilidade no $9^{\circ}$ ano de escolaridade. Podia ainda ser abordado de modo mais abrangente e ser posteriormente usado na explicação dos vários tipos de ligação química que se verificam na matéria.

\section{Estratégias Que Podem Auxiliar o Processo De Ensino e Apren- dizagem Do Conceito De Electronegatividade}

Torna-se facilmente perceptível que a estratégia de abordagem que os programas do Ministério da Educação apresentam podem dificultar a aquisição de conhecimentos por parte dos alunos e, consequentemente, contribuir para o insucesso escolar. Assim, cabe aos professores tentar colmatar estas dificuldades através de abordagens mais criativas, que fomentem uma maior compreensão dos conceitos envolvidos e potenciando a sua utilização em maior número de situações na escola e na vida diária. Sugere-se que uma das vias a seguir seja a utilização das Tecnologias de Informação e Comunicação, de forma a promover o interesse dos alunos e despertar a curiosidade pelo conhecimento científico, nomeadamente como auxiliares que permitam uma melhor visualização do que se passa a nível molecular. Nesse sentido, apresentam-se algumas sugestões que os professores poderão aplicar nas suas aulas.

Muitos alunos têm alguma dificuldade de abstracção no que se refere a conceitos em química pelo que a representação das moléculas de modo mais gráfico pode ser um auxiliar.

Os exemplos sugeridos referem-se a algumas das moléculas mais comuns do programa de Física e Química A, e permitem a visualização das suas nuvens electrónicas, do respectivo comprimento de ligação e das cargas dos iões, entre outros. Estes exemplos, que não esgotam as possibilidades, pretendem ser utilizados com os alunos de forma a estimular a sua curiosidade para o estudo da Química e facilitar o seu processo de aprendizagem.

No primeiro exemplo (Figura 2 - ver página seguinte) mostra-se uma representação gráfica que permite que os alunos visualizem a molécula de $\mathrm{HCl}$, nomeadamente, o tamanho relativo dos átomos e o comprimento de ligação.

A distância de equilíbrio e a carga de cada um dos átomos na molécula podem ser apresentados referindo que foram obtidos por um método computacional adequado.

No caso particular da molécula de $\mathrm{HCl}$, os alunos podem inclusivamente determinar o valor da diferença de electronegatividade entre os átomos de hidrogénio e de cloro, usando para tal efeito os valores registados na escala de electronegatividade de Pauling (a escala de Linus Pauling é a escala adoptada por todos os autores dos manuais escolares), ou qualquer uma das outras escalas, apresentadas neste estudo. 


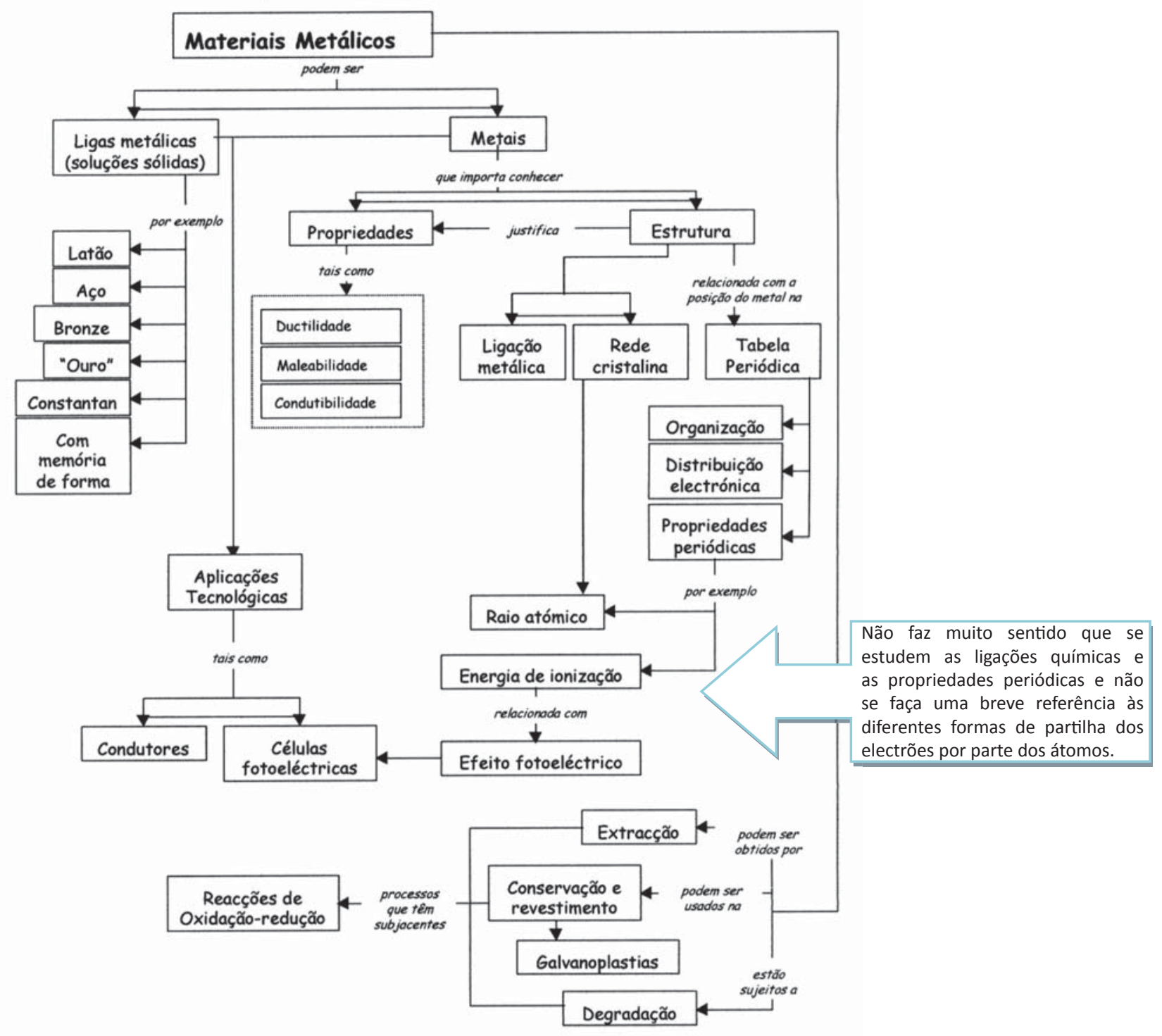

Figura 1 Esquema organizador da Unidade 1 (11ํ ano) -“Materiais e Aplicações: Metais e Ligas Metálicas " [15]

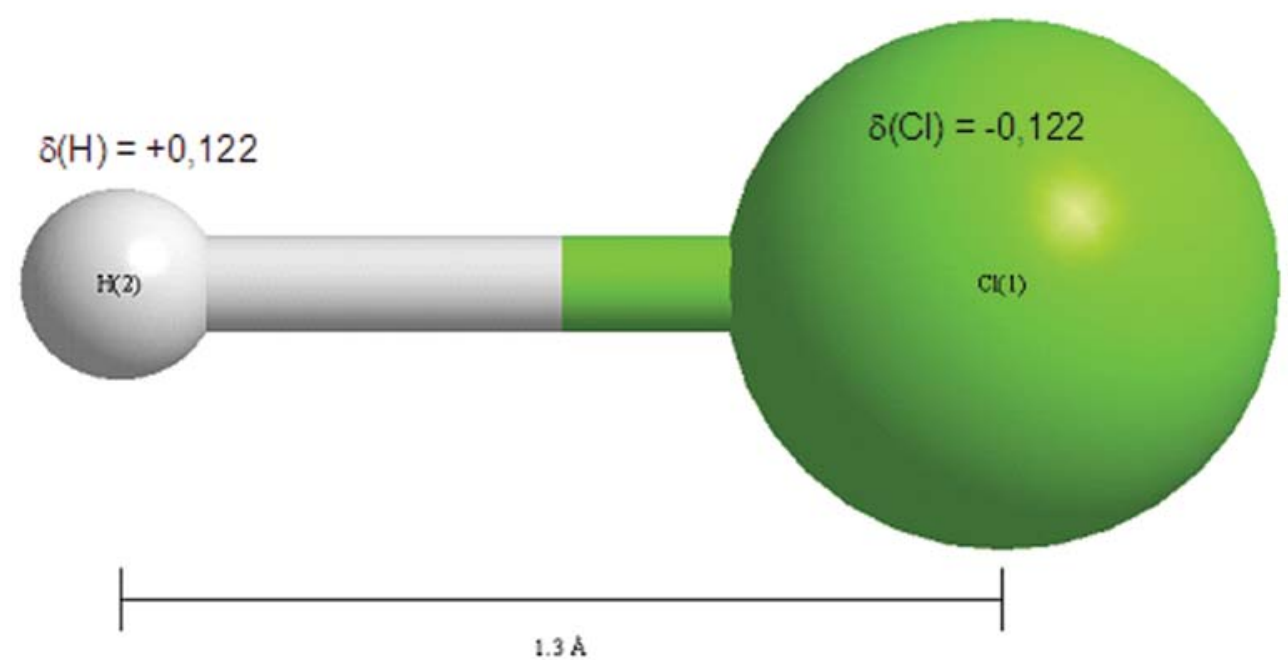

Figura 2 Imagem esquemática da molécula de ácido Clorídrico $(\mathrm{HCl}) . \delta$ representa a carga de cada um dos átomos 
Após terem efectuado este simples cálculo matemático, os alunos facilmente chegarão a um valor de diferença de electronegatividade de 0,9 (a electronegatividade é uma grandeza adimensional), pelo que se torna facilmente perceptível que um dos átomos é mais electronegativo, ou seja, tem maior tendência para atrair para si os electrões. É ainda possível relacionar este valor (equação 3) com a percentagem de carácter iónico da ligação, levando assim a uma fácil percepção do tipo de ligação existente.

Embora uma ligação só possa ser estritamente classificada como apolar quando se verifica entre dois átomos idênticos (e com vizinhanças idênticas), os critérios de classificação normalmente aceites dos vários tipos de ligação química (embora tenham essencialmente um carácter indicativo) são os seguintes:

- Diferença de electronegatividade inferior a 0,5 - ligação covalente apolar.

- Diferença de electronegatividade compreendida entre 0,5 e1,9 - ligação covalente polar.

- Diferença de electronegatividade igual ou superior a1,9 - ligação iónica.

Assim, atendendo aos critérios de classificação das ligações químicas com base na diferença de electronegatividade entre os átomos ligados, os alunos facilmente concluirão que a ligação na molécula de $\mathrm{HCl}$ é uma ligação covalente polar.
Uma outra possibilidade é representar a molécula com a sua distribuição de densidade de carga (Figura 3). Através da visualização deste tipo de imagens torna-se facilmente compreensível para os alunos, que, apesar de os átomos de hidrogénio e de cloro estarem ligados por uma ligação covalente, a partilha do par electrónico não é igual, pois os átomos têm um comportamento diferente. Em vez disso, os electrões passam em média mais tempo na vizinhança de um dos átomos. Desta "partilha desigual" do par de electrões ligante resulta um aumento da densidade de carga próximo do átomo de cloro e uma consequente diminuição de densidade de carga junto do átomo de hidrogénio, que se deve à diferente electronegatividade dos átomos. Realça-se também que

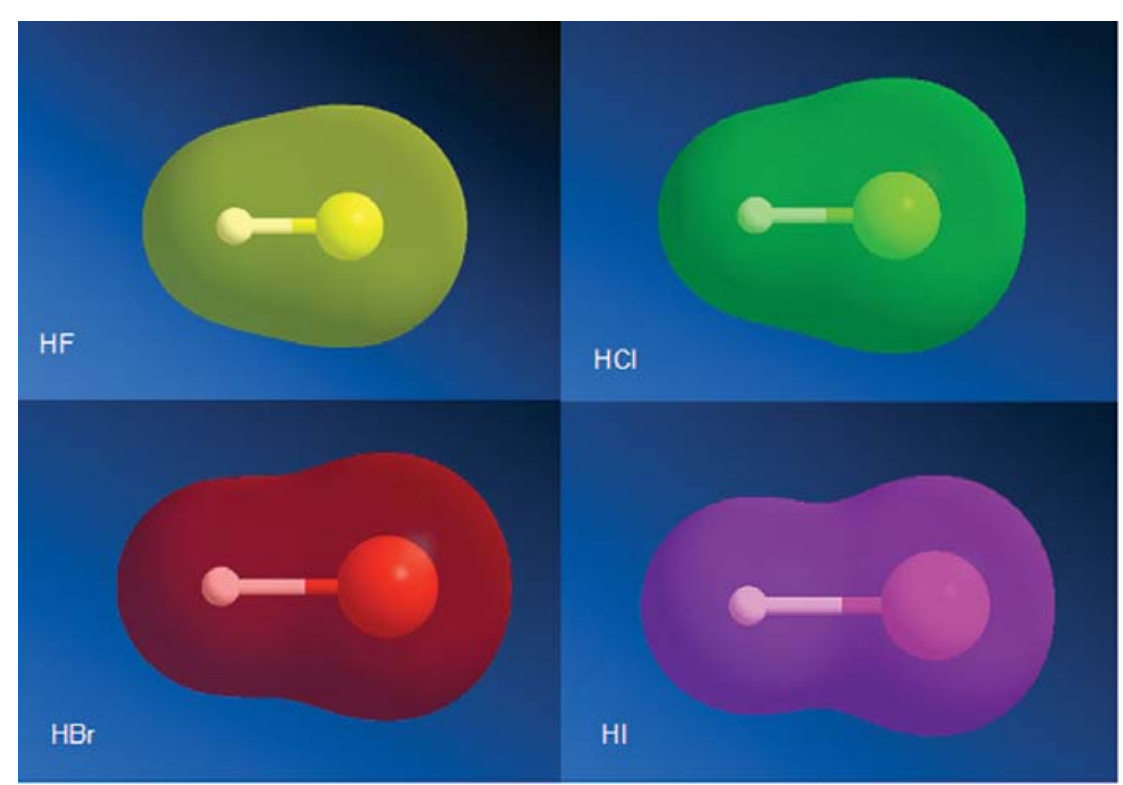

Figura 4 Distribuição da densidade de carga nas moléculas dos vários halogenetos de hidrogénio numa molécula os electrões de valência não estão localizados, mas existe uma partilha, mais ou menos desigual, dos mesmos. Esta concepção é extremamente facilitadora para a compreensão de vários conceitos de Química como, por exemplo, o conceito de ligações conjugadas e de ressonância.

Outra possibilidade, por exemplo, consiste na representação dos vários halogenetos de hidrogénio (Figura 4) e realçar como a diferença de electronegatividade entre os diferentes elementos influencia a maior ou menor polaridade da ligação bem como a influência que tem na carga que cada um dos elementos assume em cada uma das moléculas. Apresentando a carga que o hidrogénio tem em cada uma das moléculas (a do halogénio

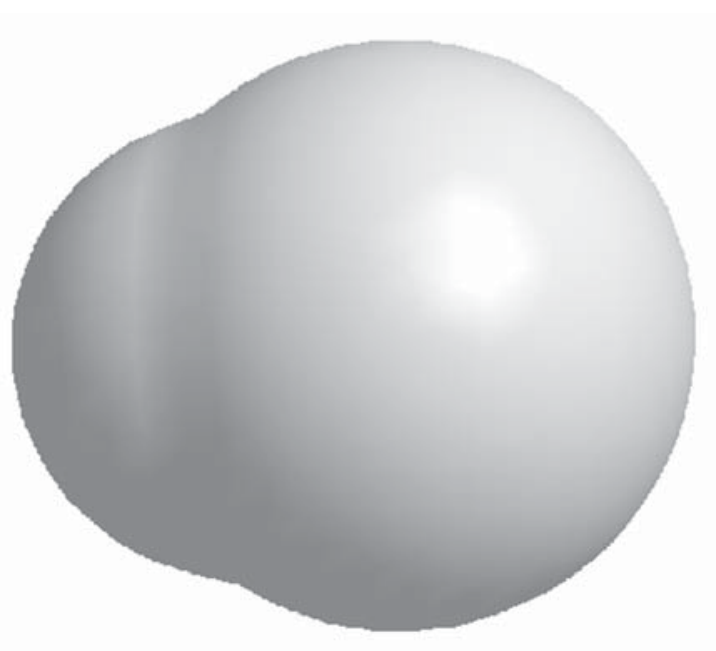

Figura 3 Distribuição da densidade de carga na molécula de ácido clorídrico será sempre o seu simétrico) vai fazer o aluno consciencializar que o mesmo elemento pode ter cargas distintas em moléculas distintas. A posterior compreensão ou explicação por parte do professor do porquê deste facto, abre as portas para um ensino em que se privilegia a apreensão dos conteúdos e não a sua memorização.

Um outro campo a explorar é a introdução do conceito de dipolo. Se o aluno perceber que o dipolo é uma grandeza vectorial que depende das cargas em jogo e da distância a que se encontram umas das outras, será mais fácil entender a maior ou menor polaridade das moléculas diatómicas. 
Uma vez introduzido este conceito os exemplos podem ser estendidos a moléculas poliatómicas. Um exemplo fácil e estimulante é a molécula do metano (Figura 5); cada uma das ligações é covalente polar $\left(\delta_{H}=+0,051\right.$; $\left.\delta_{C}=-0,206\right)$, mas a molécula como um todo é apolar. Um exemplo deste tipo pode servir para iniciar o tema da estrutura molecular. maior dificuldade em se sentirem motivados para o estudo de uma ciência que a todos os níveis é fundamental ser entendida por qualquer cidadão informado na sociedade de hoje.

É ainda de realçar que, apesar de uma grande maioria das escolas já se encontrar equipada com material informático (computadores, impres-

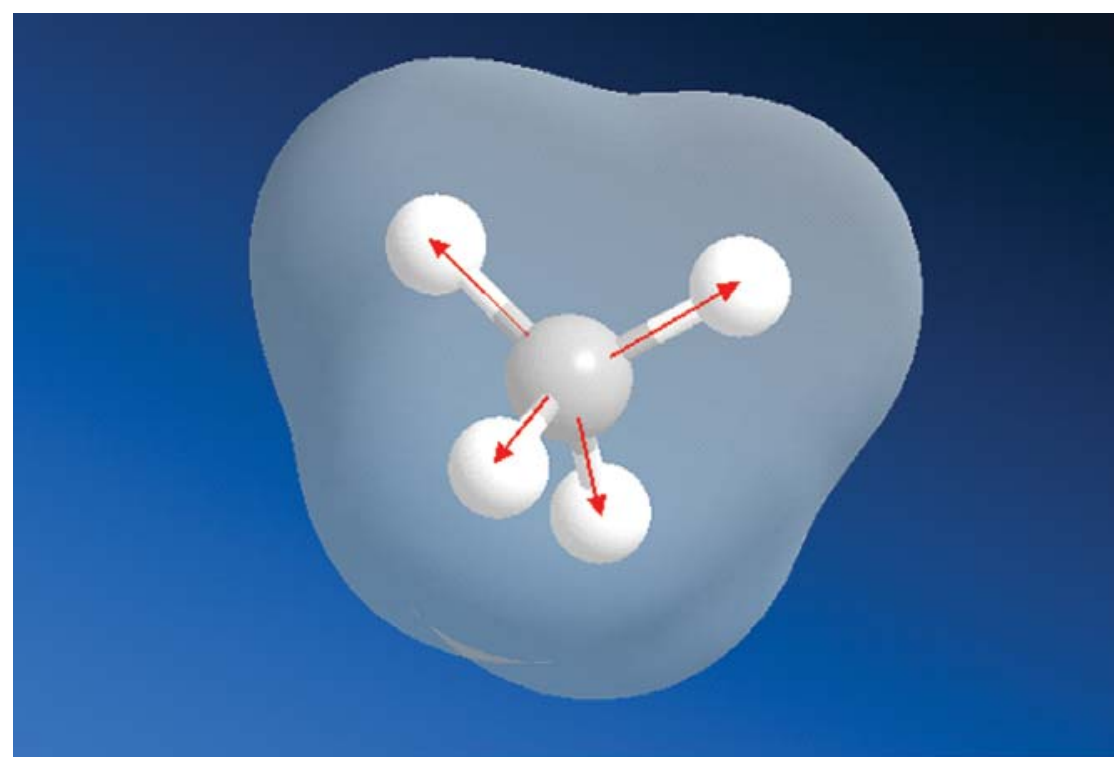

Figura 5 Distribuição da densidade de carga na molécula de metano e indicação dos dipolos a vermelho

\section{Conclusões}

No contexto de sala de aula é comum verificar que os alunos não conseguem estabelecer relações entre os vários conceitos abordados e que encaram a Química como uma área compartimentada. Esta dificuldade traduz-se, geralmente, num desinteresse crescente e, consequentemente, no insucesso escolar, tendência esta que tende a ser agravada pela incoerência do Currículo do Ensino Básico e Secundário no que diz respeito ao ensino da Química. O conceito de electronegatividade, mesmo que apresentado de forma simples, podia funcionar como o conceito aglutinador que conduzisse os alunos ao longo de todo, ou pelo menos de grande parte, do estudo desta disciplina.

De forma a melhorar o aproveitamento dos alunos seria benéfico que se procedesse, com alguma celeridade, à revisão do Currículo do Ensino Básico e Secundário no que à Química diz respeito. $\mathrm{Na}$ actual situação, é difícil vislumbrar um fio condutor e esse facto leva a que os alunos tenham uma

soras e scanners), a quantidade de software educacional, nomeadamente na área da ciência, é reduzido ou não se encontra disponível pelo facto de frequentemente ser extremamente dispendioso. Neste aspecto, o Ministério podia ter um papel importante ao negociar uma solução global para as nossas escolas junto dos vários agentes comerciais.

\section{Agradecimentos}

Os autores agradecem os comentários e sugestões do avaliador desta comunicação que muito contribuiram para a versão final apresentada.

\section{NotA}

${ }^{1}$ Apesar de $Z_{\text {eff }}$ ser habitualmente designado por carga nuclear efectiva, a designação mais correcta seria número atómico efectivo por se referir ao número de protões que efectivamente exercem um potencial atractivo sobre um electrão, depois de considerado o efeito de blindagem dos outros electrões.

\section{REFERÊNCIAS}

[1] L. Allen, "Evaluation and Test of Pauling's Electronegativity Scale", Journal of Physical Chemistry 104 (2000) 5867-5871.

[2] L. Pauling, The Nature of the Chemical Bond, Ithaca, New York: Cornell University Press, 1939.

[3] A percentagem de character iónico pode também ser calculada através da relação quadrática de Hannay e Smith

$\%$ CaracterIónico $=1-0,16\left|\chi_{A}-\chi_{B}\right|+0,35\left(\chi_{A}-\chi_{B}\right)^{2}$

Para maior detalhe ver: N. B. Hannay, C. P. Smyth, Journal of the American Chemical Society 68 (1946) 171-173.

[4] R. S. Mulliken, Journal of Chemical Physics 3 (1935) 573-585.

[5] W. Gordy, "A New Method of Determining Electronegativity from Other Atomic Properties", Physical Review A 69 (1946) 604-607.

[6] A. Walsh, Discussions Faraday Soc. 2 (1947) 18.

[7] J. Huheey, "The Electronegativity of Groups", Journal of Physical Chemistry (1965) 3284-3285.

[8] R. Sanderson, Relation of Stability Ratios to Pauling Electronegativities, Department of Chemistry, State University of lowa, lowa, 1955.

[9] A. Rochow, "A Scale of Electronegativity Based on Electrostatic Force", Journal of Inorganic and Nuclear Chemistry 5 (1958) 264-268.

[10] J. Hinze, "Electronegativity II. Bond and Orbital Electronegativities", Journal of the American Chemical Society 85(2) (1963) 148-154.

[11] L. Allen, "Electronegativity Is The Average One-Electron Energy of the Vallence-Shell Electrons in Ground-State Free Atoms", Journal of the American Chemical Society 111 (1989) 9003-9014.

[12] Para se verificar esta afirmação bastará no portal http://portalisiknowledge.com. introduzir uma pesquisa sobre "electronegativity" ou "electronegativity scales".

[13] M. Beleza, M. Cavaleiro, Ciências Físico-Químicas - Guia Prático. Lisboa, Portugal: Edições ASA, 2002.

[14]Ministério da Educação, Programa de Física e Química A - $10^{\circ}$ ou $11^{\circ}$ anos de Escolaridade, Portugal, 2001.

[15] Ministério da Educação, Programa de Física e Química A - $11^{\circ}$ ou $12^{\circ}$ anos de Escolaridade, Portugal, Março de 2003.

[16] Ministério da Educação, Programa de Química do $12^{\circ}$ ano de Escolaridade, Portugal, 2004. 


\section{Actualidades Científicas}

\section{Anetol}

Ainda a propósito da gripe $A$, circula um e-mail que afirma " $\mathrm{O}$ anis estrelado, amplamente cultivado na China, é o extracto-base (75\%) da produção do comprimido Tamiflu, da Roche. Podemos usar o nosso anis mesmo - a erva-doce - pois esta erva possui as mesmas substâncias, ou seja, o mesmo princípio activo do anis estrelado», continuando com uma exaltação das virtudes curativas da erva-doce, qual panaceia universal de acordo com o e-mail.

$\mathrm{Na}$ realidade, o anis estrelado e a erva-doce, o «nosso anis mesmo», são plantas completamente diferentes que apresentam em comum o facto de con-

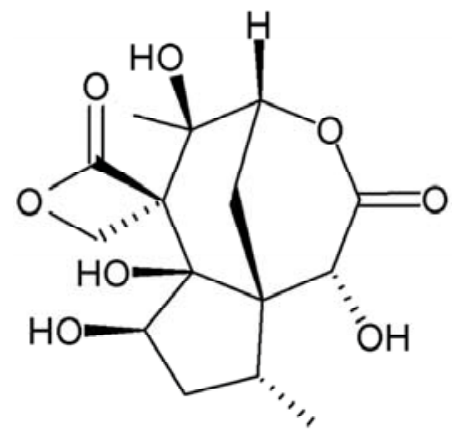

Anetol

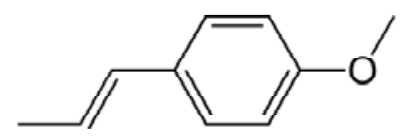

Anisatin terem grandes quantidades de anetol, o monoterpeno fenólico que Ihes confere as características organolépticas distintivas. Na realidade, também há duas espécies de anis estrelado, o chinês e o japonês, e se o primeiro pode ser utilizado sem problemas 0 segundo é extraordinariamente tóxico, ou antes, alguns dos compostos naturais que contém, como o anisatin, são mortais se consumidos em doses não muito elevadas.

O fundo de verdade nesta lenda urbana é o facto de o ácido chiquímico, um polifenol abundante no anis estrelado e ausente na erva-doce, ser um precursor do oseltamivir, conhecido comercialmente como Tamiflu, ou seja, é necessária uma série complexa de passos reaccionais para transformar este polifenol em oseltamivir.

Curiosamente, o composto que de facto ambos os anises apresentam em comum, o anetol, pode ser precursor de outro composto muito conhecido, infelizmente por outras razões: a para-metoxi-anfetamina (PMA), uma droga da família a que pertence o Ecstasy.

Palmira Silva<smiles>O=C(O)C1=CC(O)C(O)C(O)C1</smiles>

Ácido chiquímico<smiles>CCOC(=O)C1=C[C@@H](OC(CC)CC)[C@H](NC(C)=O)[C@H](N)C1</smiles>

Oseltamivir (Tamiflu)

\section{ESTRELAS DO MAR SÃO FORTES AB- SORVEDORAS DE CARBONO}

Um estudo realizado por um grupo que envolve vários centros de investigação europeus e dos EUA (Lebrato et al., ESA Ecol. Monogr., doi: 10.1890/090553 (2009)) revelou que os animais marinhos como as estrelas do mar, lírios do mar e ouriços-do-mar retêm mais carbono, sob a forma de carbonato de cálcio, do que se pensava.

Num estudo que pretende reavaliar a contribuição daqueles animais mari- nhos (equinodermes (Phylum Echinodermata)) no ciclo do carbono, estas são excelentes notícias.

O estudo envolveu a recolha de amostras de animais adultos (estrelas do mar, ouriços-do-mar e lírios do mar, entre outros), em águas superficiais e profundas a várias latitudes do Oceano Atlântico, e estimaram depois a contribuição do conteúdo em carbono de equinodermes recolhidos a várias latitudes de outros oceanos. Deste modo, estimaram a quantidade de carbonato de cálcio que os animais armazenam nos seus corpos e com que rapidez o carbono é enterrado no fundo dos mares após a morte dos animais e a sedimentação dos seus corpos.

Estimaram então que os equinodermes capturam 0,1 gigatoneladas de carbono por ano. Segundo outros investigadores, estes podem ser valores subestimados dado que há vastas regiões do Pacífico Equatorial onde existem enormes quantidades de biomassa de equinodermes que ainda não está bem estudada. 
Os equinodermes encontram-se em todos os ecossistemas, a todas as profundidades, e o seu corpo pode ser constituído por até $80 \%$ de $\mathrm{CaCO}_{3}$.

Uma questão que este estudo levanta é a do efeito da acidificação dos mares devido à absorção do dióxido de carbono atmosférico (cuja concentração está a aumentar) sobre os equinodermes e sobre a sua capacidade de reter o carbono.

Um aumento de acidez pode aumentar a dissolução dos sedimentos que derivam dos equinodermes, o que poderá ser (ou talvez já o seja actualmente) um sinal dos efeitos de acidificação.

(Adaptado de Nature News de 7 de Janeiro)

Jorge Morgado

\section{NANOMEDICINA}

Segundo um conjunto de artigos publicados recentemente (J. Phys. D: Applied Physics 42 (2009) 220301, 224001, 224002, 224303) nanopartículas magnéticas podem ser usadas para diagnosticar, monitorizar e tratar uma vasta gama de doenças e ferimentos. As nanopartículas magnéticas são usualmente produzidas a partir de magnetite comum de óxido de ferro e depois recobertas com um material biocompatível, que estabiliza e protege as nanopartículas à medida que se deslocam através do corpo. A cobertura é muitas vezes constituída por ácidos gordos. Moléculas sensoriais, corantes ou fármacos podem ser ligadas às nanopartículas através de variados grupos de ancoramento. Estas nanopartículas funcionalizadas podem ser deslocadas até um local específico usando campos magnéticos externos. Este tipo de nanopartículas têm estado a ser testadas in vivo para tratar danos no coração de ratos e para destruir, por acção do calor gerado por campos magnéticos alternados (AC), células tumorais (porque são mais sensíveis) em humanos.
Esta técnica tem sido usada para tratar, em particular, formas severas de cancro do cérebro em catorze pacientes e tem sido também usada para tratar cancro da próstata. Estes avanços têm sido conseguidos através de um significativo investimento na síntese e funcionalização das nanopartículas (adaptado de Materials Today, Dezembro de 2009).

Jorge Morgado

\section{DisPositivos ELECTRÓNICOS BIODE- GRADÁVEIS}

A medicina está evoluir no sentido de conseguirmos diagnosticar e tratar doenças in loco, ou seja, através da utilização de nanodispositivos que, circulando através da nossa corrente sanguínea, possam diagnosticar o mau funcionamento e tratar localmente as células e tecidos. Os fármacos serão assim libertados apenas no local adequado sem expor as restantes células, as quais podem ser também negativamente afectadas. Para atingir este fim, estudam-se possibilidades de usar dispositivos inteligentes, capazes de processar a informação recolhida localmente e actuar em conformidade. Os dispositivos actuais são ou implantes temporários feitos de polímeros biodegradáveis com funcionalidade electrónica limitada ou implantes permanentes feitos de silício que têm que de ser cirurgicamente removidos depois de cumprirem a sua tarefa. Pretende-se fabricar dispositivos que combinem o melhor destas duas abordagens: uso de polímeros biodegradáveis ou biocompatíveis e capazes de desempenharem funções mais complexas, como as conseguidas com os dispositivos de silício. A solução é a electrónica orgânica com base em polímeros biodegradáveis. Um passo relevante foi anunciado recentemente (Advanced Materials 21 (2009) 1). Um grupo da Universidade de Stanford (EUA) fabricou um transístor orgânico (envolvendo o uso de polímeros biodegradáveis e o uso, em muito pequenas quantidades, de ouro e prata biocompatíveis) que se mantém operacional quando exposto a ambientes aquosos, degradando-se ao fim de 50 dias. Os próximos passos serão o fabrico de outros componentes electrónicos com base em biomateriais e outros sistemas electrónicos orgânicos, que possam ser integrados em dispositivos mais complexos e implantados no corpo humano. (adaptado de Materials Today, Dezembro de 2009).

Jorge Morgado 


\section{Actualidades Científicas}

\section{NOVAS PERSPECTIVAS NO COMBATE ÀS} DOENÇAS PROVOCADAS POR AMILÓIDES

Os amilóides são depósitos de proteínas fibrosas associados a várias doenças como Alzheimer e Parkinson. Pensava-se que as fibrilas de amilóides (estruturas em forma de corda formadas por fibras de proteínas) eram inertes, mas que poderia haver fases tóxicas (que ocorriam durante a sua formação) que poderiam danificar células e provocar doenças. Contudo, num artigo publicado no passado dia 4 de Dezembro (Journal of Biological Chemistry 284 (2009) 34272) um conjunto de investigadores da Universidade de Leeds no Reino Unido, mostrou que a citotoxicidade dos amilóides aumenta coma fragmentação das fibrilas. Ou seja, são as próprias fibrilas que são tóxicas e são-no tanto mais quanto mais pequenas.

Os depósitos de amilóides podem acumular-se em muitos locais diferentes do corpo ou podem ficar localizados num órgão ou tecido particular, provocando uma gama de diferentes doenças. Esses depósitos podem, por exemplo, ser encontrados no cérebro, no caso dos pacientes que sofrem de Alzheimer ou Parkinson. O processo de formação desses depósitos (por auto-organização) é uma consequência natural da idade, já que $85 \%$ de todas as doenças causadas por depósitos de amilóides ocorrem em idades acima dos sessenta anos.

A razão porque as fibrilas amilóides mais pequenas são mais tóxicas do que as mais longas permanece por desvendar, embora se pense que as mais pequenas se infiltram mais facilmente nas células. Por desvendar permanece também o processo de formação das fibras mais pequenas: se se formam naturalmente quando se dá a auto-organização das fibrilas de amilóides ou se resultam da desagregação e fragmentação das fibras maiores (Public release de 4 de Dezembro de 2009 da Universidade de Leeds).

Jorge Morgado

\section{SínTESE MAIS FÁCIL DE ÉSTERES}

A produção de biocombustível e ésteres (usados no fabrico de fragrâncias e tecidos) pode, potencialmente, tornar-se mais verde, barata e mais eficiente, graças ao trabalho desenvolvido por um grupo da Universidade de Harvard (EUA). Estes investigadores descobriram que partículas de ouro recobertas com oxigénio podem oxidar, selectivamente, uma variedade de alcoois e aldeídos, podendo este processo ocorrer a baixas temperaturas (próximo da temperatura ambiente).
Os ésteres derivam da reacção de ácidos carboxílicos com álcoois (como metanol ou fenol). Este grupo demonstrou que o oxigénio adsorvido à superfície do ouro actua como uma base de Brønsted, provocando a cisão da ligação O-H do metanol. O intermediário adsorvido (metoxi) actua depois como um nucleófilo atacando centros deficitários electrónicos em vários aldeídos para formar ésteres metílicos.

Esta via pode oferecer alternativas mais amigas do ambiente e mais efi- cientes para a produção de ésteres de baixo peso molecular, podendo também contribuir para o desenvolvimento de fontes alternativas para produção de biocombustíveis. Falta no entanto verificar se este processo pode ser implementado em grande escala, considerando, em particular, o custo do ouro e a estabilidade do catalisador sob condições operacionais. (de Chemistry World, News, Royal Society of Chemistry, 4 de Dezembro)

Jorge Morgado 